\title{
Consenso Latinoamericano: niños pequeños para la edad gestacional
}

\author{
MARGARET CS BOGUSZEWSKI ${ }^{1}$, VERÓNICA MERICQ ${ }^{2}$, IGNACIO BERGADA $^{3}$, \\ DURVAL DAMIANI ${ }^{4}$, ALICIA BELGOROSKY ${ }^{5}$, PETER GUNCZLER ${ }^{6}$, TERESA ORTIZ ${ }^{7}$, \\ MAURICIO LLANO ${ }^{8}$, HORACIO M. DOMENÉ $^{9}$, RAÚL CALZADA-LEÓN ${ }^{10}$, \\ ARMANDO BLANCO ${ }^{11}$, MARGARITA BARRIENTOS ${ }^{12}$, PATRICIO PROCEL ${ }^{13}$, \\ ROBERTO LANES ${ }^{14}$, ORLANDO JARAMILLO ${ }^{15}$
}

1. Departamento de Pediatría, Hospital de Clínicas, Universidad Federal del Paraná, Curitiba, Paraná, Brasil.

2. Instituto de Investigaciones Materno Infantil, Facultad de Medicina, Universidad de Chile, Santiago.

3. División de Endocrinología, Hospital de Niños "Ricardo Gutiérrez", Buenos Aires, Argentina.

4. Unidad de Endocrinología Pediátrica, Instituto Infantil, Escuela de Medicina de la Universidad de São Paulo, Brasil.

5. Hospital de Pediatría Garrahan, Buenos Aires, Argentina.

6. Unidad de Endocrinología Pediátrica, Hospital de Clínicas Caracas, Caracas, Venezuela.

7. Universidad Militar Nueva Granada Hospital Militar Central, Bogotá, Colombia.

8. Departamento de Pediatría de la Universidad del Bosque, Bogotá, Colombia, Departamento de Pediatría, Centro Médico de los Andes, Fundación Santa Fe de Bogotá, Instituto de Crecimiento y Desarrollo Humanos, Bogotá, Colombia.

9. Centro de Investigaciones Endocrinológicas (CEDIE-CONICET), Hospital de Niños "Ricardo Gutiérrez," Buenos Aires, Argentina.

10.Servicio de Endocrinología, Instituto Nacional de Pediatría, Ciudad de México, México.

11. Hospital Ángeles Lomas, Ciudad de México, México.

12. Hospital Para el Niño Poblano, Puebla, México.

13.Instituto Ecuatoriano de Endocrinología y Metabolismo, IEMYR, Quito, Ecuador.

14. Unidad de Endocrinología Pediátrica, Hospital de Clínicas Caracas, Caracas, Venezuela.

15.Servicio de Endocrinología, Hospital Nacional de Niños "Dr. Carlos Sáenz Herrera", San José, Costa Rica.

Reproducción solicitada por la Rama de Endocrinología de la Sociedad Chilena de Pediatría.

Traducción al español desde su versión original en inglés, "Latin American Consensus: Children Born Small for Gestational Age" publicada en el BMC Pediatrics 2011, 11: 66 doi:10.1186/1471-2431-11-66. La versión original electrónica del artículo está disponible en: http://www.biomedcentral.com/1471-2431/11/66.

Autorizada por Gabriela Arata de Bellabarba (Editora Directora) y Mariela Paoli de Valeri (Editora de Producción) de Revista Venezolana Endocrinologia Metabolismo.

Conflicto de interés: Margaret C.S. Boguszewski es un miembro del Consejo Consultivo Estratégico KIGS; ella no tiene ninguna información financiera que declarar. Durval Daminani recibió como honorarios menos de 10000 dólares estadounidenses de la compañía Pfizer. Este estudio fue financiado por la compañía Pfizer.

Todos los demás autores declaran que no tienen conflictos de intereses.

Contribuciones de los autores: Todos los autores participaron en la reunión del consenso para discutir y votar sobre las recomendaciones. MB, VM, AB, e IB ayudaron a redactar el manuscrito. Todos los autores leyeron y aprobaron el manuscrito final.

Traducción al español: La traducción al español fue realizada por la Lcda. Iris Tommasi, bajo la asesoría de la Lcda. Milagro Ocariz, y con la colaboración de la Dra. Françoise Salager-Meyer.

Recibido el 10 de agosto de 2012, aceptado para publicación el 16 de octubre de 2012.

Correspondencia a:

Margaret CS Boguszewski

E-mail:margabogus@uol.com.br 


\section{Antecedentes}

El bajo peso al nacer $(<2500 \mathrm{~g})$ es frecuente en muchos países y representa un problema importante de salud pública que conlleva a una variedad de efectos negativos a corto y largo plazo. Mientras que cerca de la mitad de los lactantes de bajo peso en el mundo industrializado nacen prematuros $(<37$ semanas de gestación), la mayoría de ellos nacen a término en el resto del mundo ${ }^{1}$. El informe mundial de la UNICEF de $2003^{1,2}$, reveló una prevalencia global mundial de $14 \%$ de nacimientos de bajo peso, siendo mayor en Asia del Sur $(26 \%)$, del $14 \%$ en países en vía de desarrollo y $9 \%$ en Latinoamérica y el Caribe (tabla 1). Sin embargo, dos tercios de los nacimientos de algunas partes de Asia, África y Latinoamérica no son registrados porque ocurren en clínicas pequeñas o en sus domicilios ${ }^{1}$, por consiguiente, es lógico suponer que el número de nacimientos de bajo peso sea también sub-registrado.

En niños de bajo peso al nacer o PEG hay mayor probabilidad de morbilidad y mortalidad que en niños adecuados para la edad gestacional (AEG) $)^{3-5}$. Por lo tanto, es imperativo iniciar con los cuidados de la salud de estos niños tan pronto como sea posible para alcanzar resultados óptimos.

La elaboración del consenso de 2007 sobre el tratamiento de los niños nacidos PEG realizado por las Sociedades Internacionales de Endocrinología Pediátrica y la Sociedad de Investigación sobre la Hormona de Crecimiento ${ }^{3}$ incitaron a los expertos a elaborar un consenso similar para Latinoamérica. La primera reunión se llevó a cabo en Lima, Perú, en octubre de 2008, en donde tres grupos de trabajo abordaron temas claves para el debate de consenso, específicamente relacionados con la evaluación y el tratamiento de los niños nacidos PEG en Latinoamérica. En marzo de 2009, el grupo se reunió en Praga, República Checa, para discutir dichos temas y llegar a un acuerdo sobre los distintos puntos del consenso. Poco tiempo después, se elaboró este consenso, el cual se presenta aquí en su forma definitiva. Este informe presenta un resumen de los principales temas discutidos en las conferencias, en lo que se refiere a la identificación clínica y al tratamiento óptimo de los niños nacidos PEG.

\section{Discusión}

\section{Definición de niños PEG}

Aún no está clara la definición de niños PEG. La Organización Mundial de la Salud (OMS) define a los niños nacidos PEG o con restricción del crecimiento intrauterino (RCIU) como aquéllos cuyo peso al nacer está por debajo del percentil 10 por género al nacer, para la edad gestacional. Cuando los datos sobre la edad gestacional no están disponibles, el peso al nacer $<2500 \mathrm{~g}$ debería ser considerado como bajo ${ }^{6}$. Esta definición también se usa en las normas obstétricas y neonatales debido al hecho de que estos niños representan el grupo con la más alta morbilidad y mortalidad ${ }^{6-9}$. Sin embargo, los neonatos con bajo peso o talla al nacer (o ambos) con respecto a la edad gestacional deberían considerarse PEG.

Para los efectos de este consenso, un niño PEG es aquél cuyo peso y/o talla al nacer es al menos 2 desviaciones estándar (DE) por debajo de la media para su edad gestacional ${ }^{10}$.

Se recomienda particularmente que el término PEG no se utilice como sinónimo de RCIU. El término RCIU se refiere al crecimiento insuficiente del feto y solamente se debería usar si se dispone de por los menos 2 valoraciones del crecimiento intrauterino y si el feto no se está desarrollando normalmente. PEG se refiere al tamaño corporal (bajo peso y/o talla para una edad gestacional conocida) y es el usado en ausencia de información sobre el crecimiento fetal.

\section{Epidemiología}

Aunque muchos países carecen de datos sobre la verdadera incidencia de niños nacidos PEG, existen estimaciones que oscilan entre el $2,3 \%(<-2 \mathrm{DE})$ y el $10 \%(<$ del percentil 10), según la definición utilizada ${ }^{11}$. En Latinoamérica, una evaluación realizada entre 1999 y 2004 en el Hospital Militar Central de Bogotá, Colombia, reveló que el 3,6\% de 14274 recién nacidos eran PEG, definidos como $<$ del percentil 10 del peso y talla (comunicación de la 
Tabla 1. Prevalencia de bajo peso al nacer a nivel mundial por región

\begin{tabular}{|lc|}
\hline Regiones & $\begin{array}{c}\text { \% de lactantes con } \\
\text { bajo peso al nacer } \\
\text { 1995-2000 }\end{array}$ \\
\hline Asia del Sur & 26 \\
\hline África Subsahariana & 12 \\
\hline Medio Oriente y África del Norte & 11 \\
\hline Latinoamérica y El Caribe & 9 \\
\hline ECO/CEl y los Estados Bálticos & 9 \\
\hline Países menos desarrollados & 18 \\
\hline Mundo & 14 \\
\hline Países en vía de desarrollo & 14 \\
\hline Países Industrializados & 7 \\
\hline
\end{tabular}

Abreviaturas: ECO: Europa Central y Oriental; CEl: Comunidad de Estados Independientes. Fuente: Fondo de las Naciones Unidas para la Infancia (UNICEF) 2003. Informe del Estado Mundial de la Infancia. Nueva York: UNICEF, 2003. (2) Disponible en: http:// www.unicef.org/sowc03/contents/index.html. Consulta: 8 de septiembre de 2010

Dra. Teresa Ortiz). En México, una revisión de 31209 niños nacidos entre el año 2000 y 2002 mostró una prevalencia de PEG del $6 \%(\leq-2$ $\mathrm{DE}$ de peso) en la población general, desde el $6,5 \%$ al $7,2 \%$ entre trabajadores asalariados de la Ciudad de México $(\mathrm{n}=807)$ y desde el 3,7 al $6,9 \%$ en campesinos no asalariados de pequeñas comunidades rurales $(\mathrm{n}=339)^{12}$. La discrepancia entre los niños nacidos PEG en países latinoamericanos puede depender también de la gráfica de crecimiento que se utiliza, si ha sido apropiadamente actualizada y si refleja la mezcla étnica de un determinado país. Además, el número de nacimientos PEG puede depender del estrato socioeconómico y del porcentaje de malnutrición existente en los países latinoamericanos.

\section{Identificación principal}

Considerando las altas tasas de morbilidad y mortalidad en esta población, es primordial su rápida identificación. Los RN PEG tienen 5 veces más probabilidad de morir en el período neonatal y 4,7 veces más probabilidad de morir en su primer año de vida ${ }^{13}$. El nacimiento prematuro y el bajo peso al nacer son causas importantes de muerte en los países de bajos $\mathrm{y}$ medianos ingresos ${ }^{13,14}$. Los RN PEG tienen mayor riesgo de desarrollar hipertensión y diabetes tipo 2 en la edad adulta ${ }^{13,15,16}$. También presentan mayor riesgo de tener talla baja y menor coeficiente intelectual $(\mathrm{CI})^{13,15,16}$. Los niños de bajo peso al nacer con "catch up" del crecimiento temprano, tienen mayor riesgo de desarrollar obesidad en la infancia y de enfermedades del adulto, incluyendo enfermedad coronaria, accidente cerebro vascular y diabetes mellitus, las cuales, según la OMS, se encuentran entre las 10 causas principales de muerte a nivel mundial ${ }^{12,13,15,17,18}$.

La determinación precisa de la edad gestacional es esencial para el diagnóstico de PEG. E1 registro menstrual de la madre y el uso de ultrasonido, comúnmente en la semana 16 de gestación, aumenta la precisión de la estimación. Cuando esta información no está disponible, el examen físico del recién nacido mediante la escala de Ballard puede ser de gran ayuda $^{19}$. Un personal debidamente capacitado debería medir el peso, la talla y la circunferencia cefálica de acuerdo a procedimientos adecuados y estandarizados. Es de suma importancia la exactitud de las medidas del cuerpo del recién nacido. Las balanzas electrónicas para medir el peso y las cintas de papel para medir la circunferencia cefálica son métodos confiables $^{20-22}$. Esta última debería ser valorada al nacer, así como en el primer control pediátrico durante el primer mes de vida con el propósito de obtener una medida más exacta. La medición de la talla de un lactante puede ser poco confiable ${ }^{20,23}$, pero su precisión puede mejorar si es medido por 2 personas utilizando un estadiómetro apropiado ${ }^{20,24}$. Los valores deberían compararse con las gráficas de referencia de una población específica, permitiendo clasificar el niño como PEG o AEG según la definición elegida ${ }^{25}$. Son preferibles los gráficos referenciales específicos de cada país por tamaño al nacer ${ }^{26}$, pero en algunos países latinoamericanos estos gráficos aún no están disponibles. Es necesario elaborar estos gráficos referenciales de tamaño al nacer en cada país, ya que la definición de PEG podría ser errónea en algunas áreas.

Las gráficas de crecimiento específicas para Argentina se han usado por más de 2 décadas $^{27,28}$. En el 2009, Lejarraga y colaborado- 
res publicaron actualizaciones de estos gráficos recalculando los percentiles y los valores Lambda-Mu-Sigma (LMS) desde el nacimiento hasta la madurez e incorporando datos actualizados de la OMS desde el nacimiento hasta la edad de 2 años ${ }^{29}$. Los gráficos de crecimiento de Argentina están disponibles en la dirección URL: http://www.garrahan.gov.ar/ tdecrecimiento. Se recomiendan los gráficos de crecimiento de Babson y Benda actualizados en el 2003 cuando no se dispone de una referencia nacional para el crecimiento de niños prematuros y el tamaño al nacer ${ }^{20,30}$. Los niños nacidos prematuramente $(<37$ semanas de edad gestacional) deberían ser evaluados considerando sus características especiales. Entre ellas estarán los recién nacidos PEG y AEG y se deben usar referencias apropiadas para los prematuros ${ }^{20}$. Sin embargo, es importante reconocer que la definición de PEG en lactantes prematuros puede ser difícil ya que los gráficos referenciales de pre término típicamente no incluyen a los lactantes extremadamente prematuros. Cada país debería hacer un esfuerzo para recopilar los gráficos de crecimiento que representen una gran cantidad de lactantes prematuros de diferentes edades gestacionales para tener un conjunto de datos más completo.

\section{Causas del diagnóstico de PEG}

Muchos factores de riesgo relacionados con el bajo peso al nacer se superponen de manera significativa al factor de riesgo relacionado con lactantes nacidos prematuramente. La caracterización de un lactante PEG debería tomar en cuenta la talla, peso, paridad, edad, etnicidad y ubicación geográfica de la madre. Se debería también determinar la malnutrición materna (aumento insuficiente del peso durante la gestación), tamaño y disfunción de la placenta y la presencia de enfermedades de la madre $^{11}$. El tabaquismo, el consumo de alcohol y el uso de drogas son causas evitables de RCIU, por lo tanto, se deberían registrar los hábitos de la madre relacionados con estos factores. El crecimiento fetal depende tanto de los factores genéticos como de un ambiente óptimo de salud materno-fetal que permita el flujo libre de los nutrientes y el oxígeno, además de la integridad de los factores de crecimiento similares a la insulina (IGF-1 e IGF-2), la acción y síntesis de la insulina. Adicionalmente, un exceso de cortisol en el sistema circulatorio fetal produce una alteración en el crecimiento del mismo.

\section{PEG y crecimiento}

\section{Seguimiento}

La mayoría de los niños nacidos PEG recuperan el déficit de peso y talla. Los RN a término PEG generalmente completan el "catch up" alrededor de los 2 años de edad ${ }^{31-33}$, mientras que los $\mathrm{RN}$ prematuros pueden tardar más en completar dicho "catch up" que los RN a término $^{34}$. La recuperación se completa cuando ellos alcanzan su potencial genético que está determinado por la estatura parental ${ }^{33}$. Sin embargo, entre el 10 y el 15\% de esos RN PEG continuará presentando una talla significativamente menor $(\leq-2 \mathrm{DE})$ durante la infancia y la vida adulta ${ }^{31,35,36}$.

Aproximadamente el 90\% de los niños nacidos PEG a término y sanos, experimentarán recuperación del retardo del crecimiento durante sus primeros 2 años de vida ${ }^{31}$, lo cual puede ocurrir a una edad tan temprana como a las 12 semanas de edad postnatal ${ }^{37}$. Por lo tanto, este consenso recomienda que los niños nacidos PEG debieran ser evaluados cada 3 meses durante el primer año de vida y cada 6 meses durante el segundo. En cada cita se debería medir peso, talla y circunferencia cefálica. Un niño que no presente recuperación del crecimiento durante los 6 primeros meses de vida se debería evaluar por más tiempo. La misma recomendación es válida para un niño cuyo peso es $\leq-2 \mathrm{DE}$ a la edad de 2 años. En estos casos, se deberían descartar las enfermedades pediátricas comunes, los trastornos genéticos y las disfunciones hipotalámicas y/o de la pituitaria. Los niños PEG que no recuperan la talla generalmente tienen una secreción endógena de hormona de crecimiento adecuada en respuesta a las pruebas farmacológicas. Sin embargo, a menudo tienen niveles séricos bajos de IGF-1 y una alteración en los patrones de secreción fisiológica de hormona de crecimiento ${ }^{32,38}$. 
Idealmente, el diagnóstico PEG implica datos de referencia de talla y peso en una población geográfica cuya etnicidad se conoce. Si los gráficos aprobados por las Asociaciones Pediátricas locales no están disponibles, desde el nacimiento hasta la edad de 5 años, se podrían utilizar los gráficos de crecimiento de la OMS (www.who.int/childgrowth) ${ }^{39}$. Los gráficos de la OMS combinan datos de un seguimiento longitudinal desde el nacimiento hasta los 24 meses y una encuesta transversal de niños de edades comprendidas entre $18 \mathrm{y}$ 71 meses. También se incluyeron los lactantes alimentados con pecho y los niños provenientes de Brasil, Ghana, India, Noruega, Omán y los Estados Unidos. Después de los 5 años de edad se recomienda la Referencia de la OMS de 2007 que es una actualización del Centro Nacional para la Estadística de Salud (CNES) de 1997/referencia OMS, que utilizó el conjunto de datos originales del CNES con datos de los estándares de crecimiento infantil de la OMS (www.who.int/growthref/en/) ${ }^{40}$.

\section{Criterios para el inicio del tratamiento con hormona de crecimiento}

\section{Edad}

En julio de 2001, la Agencia Federal para el control de Alimentos y Fármacos (FDA) aprobó el tratamiento con la hormona de crecimiento (GH) (inyección de $\mathrm{GH}$ [de origen $\mathrm{ADNr}]$ ) para el tratamiento prolongado del déficit de crecimiento en niños nacidos PEG, que no experimentaron catch-up del crecimiento a la edad de 2 años ${ }^{10,41,42}$. Esto se refiere a los niños mayores de 2 años de edad con talla baja $(<-2 \mathrm{DE})$, que presentaron un bajo peso al nacer $<2500 \mathrm{~g}$ a una edad gestacional de $\geq 37$ semanas $^{41}$, o bien, un peso o talla al nacer menor al percentil 3 para la edad gestacional ${ }^{41}$, o un índice de peso ponderado $(100 \times$ [peso en $\left.\mathrm{g}] /[\text { talla en } \mathrm{cm}]^{3}\right)<-2 \mathrm{DE}$.

En Europa, en junio de 2003 fue aprobado el tratamiento con hormona de crecimiento por el Comité de Especialidades Farmacéuticas (CPMP) para niños nacidos PEG (peso y talla al nacer $<-2 \mathrm{DE})$ con talla baja $(<-2,5 \mathrm{DE}$ y/o talla $<-1,0 \mathrm{DE}$ del potencial genético) y para aquellos que no completen el catch-up del cre- cimiento a la edad de 4 años o más ${ }^{43}$.

Argente y colaboradores analizaron recientemente los resultados de niños PEG entre 2-5 años de edad tratados con $\mathrm{GH}^{44}$. Se reportó un mayor aumento en la velocidad de crecimiento en el grupo de niños menores de 4 años de edad. Por lo tanto, se debería considerar iniciar el tratamiento poco después de los 2 años de edad, particularmente cuando la velocidad de crecimiento está por debajo del percentil 25 para la edad.

\section{Recomendaciones para la dosificación con la hormona de crecimiento}

Se ha observado un aumento notorio en la velocidad de crecimiento dependiente de la dosis durante el primer año de tratamiento en los niños nacidos $\mathrm{PEG}^{10,45}$. La talla óptima se obtiene con un tratamiento prolongado con $\mathrm{GH}$ antes de comenzar la pubertad ${ }^{46}$. Además, la ganancia de talla obtenida con el tratamiento durante los años previos a la pubertad se mantiene hasta la talla final ${ }^{46}$. Las recomendaciones de las dosis de GH para niños PEG difieren en los Estados Unidos y Europa: la dosis recomendada en los Estados Unidos es hasta $0,48 \mathrm{mg} / \mathrm{kg} / \mathrm{semana}(68,5 \mu \mathrm{g} / \mathrm{kg} /$ día $)$ dividida en dosis diarias ${ }^{41}$, y la dosificación correspondiente en Europa es de 0,035 mg/kg/día hasta que se alcance la talla definitiva ${ }^{43}$. Nosotros recomendamos una dosis inicial de $\mathrm{GH}$ de $0,33 \mathrm{mg} / \mathrm{kg} / \mathrm{semana}(\approx 47 \mu \mathrm{g} / \mathrm{kg} /$ día o $0,15 \mathrm{UI} /$ $\mathrm{kg} /$ día) con ajustes de dosis basados en la ganancia de peso para aquellos niños con talla $<-2$ y $>-3$ DE. Sin embargo, para aquellos niños con talla $<-3 \mathrm{DE}$, cuando se desea la recuperación rápida del crecimiento, se podría indicar una dosis más alta $(0,48 \mathrm{mg} / \mathrm{kg} / \mathrm{sema}-$ na) sin ajuste de dosis basado en el peso, hasta que alcancen la dosis regular de $0,33 \mathrm{mg} / \mathrm{kg} /$ semana y niveles de IGF-1 dentro del rango superior normal. Una dosis mayor de $\mathrm{GH}$ al inicio del tratamiento, logrará un catch-up del crecimiento más rápido en aquellos niños que están más seriamente comprometidos.

\section{Evaluación inicial y seguimiento durante el tratamiento con hormona de crecimiento}

Los estudios iniciales incluyendo las determinaciones hormonales (tiroides, IGF-1) y 
metabólicas (glucemia, insulina y perfil lipídico) son obligatorios antes de iniciar el tratamiento con GH. Se recomienda el seguimiento cuidadoso durante el tratamiento con esta hormona. Un médico experimentado en el uso de la GH debería evaluar al niño cada 3 a 6 meses (examen físico y evaluación de laboratorio) con el fin de determinar si se debe o no ajustar la dosis ${ }^{47}$. Se debería monitorear glucemia, función tiroidea, HbAlc e IGF-1 una vez al año, excepto en casos que muestren clara evidencia clínica de resistencia a la insulina o una $\mathrm{HbA} 1 \mathrm{C}$ de $6 \%$ al inicio del tratamiento. Aunque es necesario medir los niveles de IGF-1 una vez al año, es preferible hacerlo dos veces al año. El monitoreo de los cambios en los niveles basales de insulina y de los sustitutos de la sensibilidad a la insulina también son útiles en el seguimiento de estos niños ${ }^{48}$. Particularmente, los efectos adversos relacionados con la GH no son más comunes en los niños PEG que en otras condiciones tratadas con dicha hormona ${ }^{3}$. Los pacientes con una fuerte carga de historia familiar de diabetes tipo 2, se deben evaluar con una prueba de tolerancia oral a la glucosa (PTOG) al inicio y luego cuando sea apropiado; continuar con valores de laboratorio cada 3 a 6 meses.

\section{Criterios para interrumpir el tratamiento con hormona de crecimiento}

Se debe continuar el tratamiento con $\mathrm{GH}$ si durante el primer año se observa una respuesta positiva del crecimiento (velocidad de crecimiento $>+0,5 \mathrm{DE})^{3}$. Se interrumpe el tratamiento en la adolescencia si la velocidad de

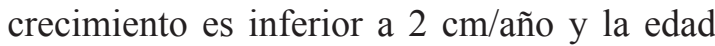
ósea es $>14$ años para las niñas y $>16$ años para los niños, lo cual corresponde al cierre de las placas de crecimiento epifisiario ${ }^{43}$.

\section{Efectos adversos}

La terapia continua con GH no está relacionada con efectos adversos graves en niños de talla baja nacidos $\mathrm{PEG}^{49-51}$. No obstante, debido al aumento de la prevalencia de trastornos metabólicos e hipertensión arterial en adultos nacidos $\mathrm{PEG}^{15,17,18,52-55}$, se debe prestar atención específica al metabolismo de la glucosa y la ganancia de peso en estos niños. Estudios previos han demostrado que con la interrupción de un tratamiento a largo plazo con $\mathrm{GH}$ en adolescentes PEG, se normalizaron los niveles de insulina (tanto en ayunas como estimulada) después de haber presentado un aumento significativo durante la terapia con esta hormo$\mathrm{na}^{56,57}$

El tratamiento con $\mathrm{GH}$ no parece estar relacionado con un incremento del riesgo de malignidad ${ }^{57}$. Se recomienda medir las concentraciones de IGF-1, y reducir la dosis de GH cuando la IGF-1 plasmática se encuentre por encima de $+2 \mathrm{DE}^{58,59}$. En un estudio de seguimiento a largo plazo de la terapia con $\mathrm{GH}$ en niños de talla baja nacidos PEG, los niveles altos de IGF-1 fueron completamente revertidos luego de la interrupción del tratamiento ${ }^{60}$.

\section{PEG y pubertad}

\section{Crecimiento puberal}

Los datos actuales relacionados con el inicio, tiempo, duración y progresión de la pubertad en niños nacidos PEG, son limitados y difíciles de comparar debido a las diferencias metodológicas disponibles en la literatura médica (por ejemplo: diferencias en la definición de PEG, PEG con o sin recuperación del crecimiento). Algunos estudios muestran un desarrollo puberal normal pero temprano, sin embargo, otros señalan un comienzo puberal $\operatorname{tardío}^{61-63}$.

En modelos humanos, se observó una reducción en el crecimiento puberal ${ }^{64}$. En modelos femeninos, se reportó un retardo del crecimiento de aproximadamente $4 \mathrm{~cm}^{65}$. Este hallazgo sugiere que hay susceptibilidades relacionadas a dimorfismo de género para los cambios puberales postnatales de los niños PEG $^{62,65}$.

En relación con la edad del inicio de la pubertad, la mayoría de los estudios indican una edad similar a aquella de los niños nacidos $\mathrm{AEG}^{62}$; sin embargo, varios investigadores han mostrado bien sea pubertad temprana ${ }^{61,65-67}, \mathrm{o}$ tardía $^{68,69}$. En un estudio con animales, los investigadores postularon que un mayor nivel de exposición a la insulina en la vida postnatal de aquellos nacidos PEG con ganancia acelerada de peso, puede inducir una secreción precoz y 
exagerada de LH que conlleva a un desarrollo puberal temprano ${ }^{70}$.

También se ha señalado la edad de la menarquia como temprana o dentro del rango normal ${ }^{62,64,66,71-75}$. Las niñas nacidas PEG que experimentan una ganancia rápida de peso en los primeros meses de la infancia tienen más probabilidades de presentar adrenarquia prematura. Estas niñas pueden tener una pubertad y una menarquia más temprana que las niñas AEG con adrenarquia prematura ${ }^{73,76}$.

Aunque la edad ósea se utiliza para evaluar la maduración esquelética, se ha sugerido que el uso de la misma para el pronóstico de la talla final es de poco valor en los niños nacidos PEG, y por lo tanto, se debería usar con precaución $^{77}$.

Los niños PEG que no recuperan el crecimiento y que son tratados con $\mathrm{GH}$ tienen un inicio y progresión de la pubertad normales ${ }^{68}$. Sin embargo, el uso tardío de dicha hormona, poco tiempo antes o después del inicio de la pubertad, no mejora la talla final de manera significativa.

\section{Función gonadal femenina}

Las niñas nacidas PEG con recuperación del crecimiento presentan menor sensibilidad a la insulina, y existen datos que muestran una mayor incidencia de hiperandrogenismo adrenal y ovárico clínicamente evidente por pubarquia precoz ${ }^{76,78,79}$. No obstante, estos datos fueron recolectados en una población seleccionada de una clínica endocrina ${ }^{76,78,79}$; otros estudios no han confirmado dichas relaciones ${ }^{80-84}$. Hasta ahora, no hay datos suficientes para confirmar alteración en la función ovárica, en la fertilidad o una menopausia precoz en estas niñas.

\section{Uso de la metformina}

Un informe preliminar sugiere que el uso precoz de la metformina en una edad perimenarquial en niñas nacidas con bajo peso y con una historia de pubarquia precoz previene la progresión al Síndrome de Ovarios Poliquísticos (SOP), mejora la sensibilidad a la insulina y normaliza la composición corporal, los perfiles lipídicos y la secreción de la $\mathrm{GH}^{85}$. Un informe posterior realizado por el mismo grupo en niñas nacidas con bajo peso y pubarquia precoz, el tratamiento con metformina estuvo relacionado con una composición corporal menos adiposa, un retardo de 0,4 años en el inicio clínico de la pubertad, un retraso de al menos 1 año en el aumento de IGF-1 circulante relacionado con la pubertad, y una estabilidad en la ganancia de peso $^{86}$. Estos hallazgos aún deben ser confirmados por otros estudios, y por lo tanto, se consideran todavía preliminares.

\section{Función gonadal masculina}

Poco se sabe acerca de los efectos a largo plazo del RCIU o de los nacidos PEG y su relación con la función hipotálamo-hipófisisgonada en varones ${ }^{87}$. Se ha relacionado el bajo peso al nacer con un aumento en la frecuencia de hipospadia, criptorquidia y cáncer testicu$\operatorname{lar}^{87}$. Este conjunto se conoce como Síndrome de Disgenesia Testicular y se ha propuesto un origen fetal común para esta relación ${ }^{87-91}$. No existen datos suficientes acerca de la calidad del semen y de su relación con la recuperación del crecimiento o el tratamiento con GH en estos individuos. Además, se han reportado algunas diferencias en los niveles de las hormonas pituitarias y testiculares, y en los volúmenes testiculares ${ }^{92}$. Sin embargo, no hay consenso en este aspecto y no hay tampoco distinción entre los individuos que tuvieron criptorquidia ${ }^{93}$. Se necesita llevar a cabo estudios adicionales con el fin de evaluar el impacto del RCIU y PEG sobre el eje hipotálamo-hipófisis-testículo.

\section{PEG y riesgos metabólicos}

Se ha relacionado un aumento significativo del riesgo de desarrollar enfermedades cardiovasculares (hipertensión, dislipidemia) y diabetes tipo 2 en el adulto con bajo peso al nacer ${ }^{52-54,94}$. Se formularon tres hipótesis principales para explicar la relación entre el bajo peso al nacer y el incremento de los riesgos metabólicos. La primera hipótesis, del cortisol fetal, propuso que la restricción de los nutrientes maternos puede actuar para reprogramar el desarrollo del eje hipófiso-adrenal, lo cual resulta en una exposición excesiva a los glucocorticoides y resultados adversos para la salud en etapas posteriores de la vida. En esta hipótesis, la 11-beta-hidroxiesteroide-deshidroge- 
nasa (11 beta-HED) desempeña un papel clave al convertir el cortisol activo en cortisona inactiva. Esta enzima resguarda al feto de los efectos retardantes del crecimiento de los glucocorticoides maternos ${ }^{95-97}$.

La segunda hipótesis es la de la insulina fetal, propone que la resistencia a la insulina determinada genéticamente resulta en la alteración del crecimiento mediado por la insulina en el feto, así como resistencia a la insulina en la vida adulta ${ }^{98}$. Hay evidencia que sustenta esta hipótesis en una minoría de casos de bajo peso al nacer. Por ejemplo, las enfermedades monogénicas con deterioro en el sensor de la glucosa, disminución de la secreción de la insulina o aumento de la resistencia a la misma, están relacionadas con una alteración del crecimiento fetal.

La explicación más plausible de esta relación, sin embargo, es la tercera hipótesis, del catch-up del crecimiento. Los niños PEG pueden presentar una disminución en la sensibilidad a la insulina precozmente en la vida ${ }^{15,99-101}$. En el primer estudio para evaluar la secreción y la sensibilidad a la insulina tanto en los niños PEG como en los AEG desde el nacimiento hasta la edad de 1 año $^{99}$, los investigadores de la Universidad de Chile, Santiago, informaron que para la edad de 1 año, los niños PEG con recuperación de la ganancia del peso (es decir, ganancia de peso con puntaje de $\mathrm{DE}>0,67)$ tenían mayores niveles de insulina en ayunas y resistencia a la misma (área de la insulina por debajo de la curva durante la glucosa IV) que los niños AEG. En un estudio de seguimiento de estos pacientes, los investigadores reportaron que la DE de ganancia en peso continuó hasta la edad de 3 años en los niños nacidos PEG, y la resistencia a la insulina también progresó durante este período ${ }^{15}$. A los 3 años de edad, no se observaron diferencias en el peso o el Índice de Masa Corporal entre estos niños PEG y AEG, tampoco hubo diferencias entre los grupos en la primera fase de secreción de insulina. No obstante, los niños PEG tuvieron un menor índice de disposición de glucosa (menor compensación de células beta) que persistió hasta después de la ganancia postnatal de peso. Dichos niños también manifestaron una marcada transición desde menor insulina en ayunas y mayor sensibilidad a la insulina al nacer, hasta resistencia a la insulina a la edad de los 3 años. Los investigadores observaron que esta transición se relacionaba con una recuperación postnatal rápida del peso ${ }^{15}$, la cual puede estar relacionada con una mayor tendencia a la deposición de la grasa central. Ellos recomendaron el monitoreo prolongado de la homeostasis de la glucosa en todos los niños PEG, independientemente de la recuperación postnatal del crecimiento. Por lo tanto, no se debería permitir ganar peso muy rápidamente o excesivamente a los niños nacidos PEG en un esfuerzo por evitar el desarrollo de trastornos metabólicos.

Los mecanismos fisiopatológicos de la resistencia a la insulina son posiblemente un factor secundario de una deficiencia nutricional prolongada relativa en el feto. Durante dicho período el metabolismo fetal se reajusta constantemente al crecimiento lento con una resistencia relativa a la insulina, IGF-1, y la GH. Cuando esta "adaptación" es inconsistente con la nutrición postnatal, puede asociarse a una ganancia rápida de peso durante la infancia, y puede resultar en el desarrollo de un conjunto de signos del síndrome metabólico ${ }^{55,102}$, con resistencia a la insulina como factor clave. Este conjunto aumenta el riesgo de experimentar comorbilidades, tales como obesidad, diabetes, dislipidemia, enfermedad coronaria, e hipertensión, entre otras ${ }^{103,104}$. De hecho, la sumatoria de factores de riesgo (por ejemplo: ganancia rápida de peso, historia familiar y grupo étnico) contribuyen a exacerbar el riesgo de síndrome metabólico en los niños nacidos PEG. En particular, estos niños no necesariamente tienen sobrepeso o son obesos, pero tienen una mayor composición corporal "adiposa" que contribuye a los rasgos metabólicos ${ }^{55}$. En estos casos, la detección de los signos clínicos de la resistencia a la insulina, tal como la acantosis nigricans, es de suma importancia. Además, el monitoreo periódico de la presión sanguínea es vital, especialmente si tienen sobrepeso o son obesos.

La eficacia del tratamiento con $\mathrm{GH}$ en niños nacidos PEG se ha comprobado en varias series y por resultados en la talla final bajo tratamiento continuo con dicha hormona ${ }^{3,44,46,51,57,105}$. Sin 
embargo, conociendo el efecto de la GH sobre el metabolismo de la glucosa, la seguridad en los niños PEG tratados con GH en relación al metabolismo de la glucosa, ha sido y continúa siendo un tema de particular importancia. Los niños PEG tratados con $\mathrm{GH}$ experimentan un aumento transitorio en las concentraciones de insulina sérica y grados variables de compromiso en la tolerancia a la glucosa ${ }^{106}$. Investigaciones previas han señalado estos efectos colaterales, los cuales son el resultado de una respuesta predecible a las acciones fisiológicas de la $\mathrm{GH}^{51,107-110}$. No obstante, no se han observado efectos a largo plazo sobre la prevalencia de diabetes tipo $2^{107}$. Además, los efectos de la resistencia a la insulina parecen ser reversibles $^{56,108,111,112}$.

Por del hecho de que un alto porcentaje de individuos con intolerancia a la glucosa presenta un nivel de glucemia en ayunas por debajo de $100 \mathrm{mg} / \mathrm{dL}$, es aplicable la prueba de tolerancia a la Glucosa Oral (TGO) en niños y adolescentes con factores de riesgo tales como: obesidad, historia familiar, e hipertensión. Sin embargo, no recomendamos que se indique esta como un examen complementario general para niños nacidos PEG sino que debería individualizarse para niños con ganancia rápida de peso, acantosis nigricans, dislipidemia (triglicéridos altos) o una historia familiar importante de síndrome metabólico ${ }^{9}$.

También se reportaron mejorías en el perfil lipídico y la presión sanguínea relacionados con el síndrome metabólico en niños PEG que reciben tratamiento con $\mathrm{GH}^{56}$. Es importante determinar las concentraciones del colesterol total, cHDL y cLDL, y especialmente los triglicéridos plasmáticos. Todos estos valores deben compararse con estándares apropiados para niños de diferentes edades.

\section{Implementación}

Para la identificación de PEG, es importante el uso de datos de referencia de peso y talla al nacer, específicos para cada población, etnia o país, si se encuentran disponibles. No obstante, las mediciones exactas de peso, talla, circunferencia cefálica y particularmente, datos gestacionales precisos, son esenciales para un diagnóstico correcto de $\mathrm{PEG}^{10}$. En casos de sospecha de diagnóstico de PEG, los pediatras deberían obtener información de los datos ultrasonográficos, si se realizaron durante el embarazo, así como considerar también obtener otros datos sobre el embarazo, perintatales/ postnatales inmediatos y datos de crecimiento postnatal precoz, si están disponibles ${ }^{10}$. Es importante, si es posible, la identificación de la causa del diagnóstico de PEG, por ejemplo factores fetales [anomalías congénitas y genéticas], factores maternos, factores nutricionales o de abuso de sustancias, factores uterino/placentarios, factores demográficos, y gestación múltiple, ya que puede afectar el tratamiento. En los niños que no experimentan catch-up del crecimiento, debe realizarse un seguimiento físico y de laboratorio a largo plazo, por un endocrinólogo o un pediatra con experiencia en endocrinología. Si fuese necesario, ellos se podrían someter temprano a una terapia con $\mathrm{GH}^{3}$.

Los obstetras y los ginecólogos deberían referir los embarazos con diagnósticos de RCIU a una clínica obstétrica de alto riesgo con cuidado especial de algunas enfermedades (por ejemplo: disfunción plaquetaria, hipertensión) con el fin de reducir la gravedad del RCIU y la frecuencia de niños nacidos PEG. Aunque las clínicas de alto riesgo están usualmente localizadas en grandes ciudades, las organizaciones de salud pública deberían estar conscientes de las necesidades de estos pacientes y desarrollar redes especiales para ellos. Los neonatólogos deberían estar alertas de las posibles consecuencias del estrés fetal agudo que puede ocurrir durante el trabajo de parto y/o durante el parto por una cesárea de un lactante PEG, además de todas las complicaciones conocidas como es el caso del distrés respiratorio, hipoglicemia e hipokalemia.

El próximo paso importante es un programa para la difusión médica y el entrenamiento de miembros de un equipo multidisciplinario. Los pediatras y neonatólogos deben proveer el adiestramiento en el manejo adecuado de los recién nacidos prematuros y a término PEG. Las complicaciones inmediatas, mediatas y a largo plazo se deben discutir haciendo énfasis en los problemas de crecimiento y las alteraciones metabólicas que pueden desarrollarse. Los pediatras deberían compartir su conoci- 
miento a través de talleres multidisciplinarios. Además, sería importante proveer materiales escritos sobre el monitoreo del adecuado crecimiento, la fase de recuperación, y el período de seguimiento, con el propósito de detectar alteraciones metabólicas y de crecimiento en estos pacientes. Para el adecuado examen y tratamiento de alteraciones metabólicas y de crecimiento en aquellos niños nacidos PEG, se deben desarrollar políticas que incluyan el cuidado médico primario y pediátrico, así como la referencia a un endocrinólogo pediatra.

\section{Resumen}

La mayoría de los niños nacidos PEG se recuperan del déficit de peso y talla. Sin embargo, del 10 al 15\% de los niños nacidos PEG continuarán teniendo talla baja. El tratamiento con $\mathrm{GH}$ beneficia el potencial de crecimiento en los niños nacidos PEG de baja talla. En ambos sexos, se debe tomar en cuenta la función gonadal especialmente durante la pubertad. Se debe monitorear el catch-up excesivo de talla, y particularmente de peso, en niños con restricción del crecimiento fetal. La ganancia excesiva de peso está relacionada muy frecuentemente con riesgo metabólico más tarde en la vida. Finalmente, un grupo multidisciplinario que incluya perinatólogos, pediatras, nutricionistas, y endocrinólogos pediatras deben seguir a los niños nacidos PEG con el fin de mejorar el crecimiento, la homeostasis de la glucosa y la función gonadal.

\section{Resumen de las guías del Consenso Latinoamericano sobre el diagnóstico de PEG}

- Se recomiendan los gráficos aprobados por las Asociaciones Pediátricas locales. Si estos no están disponibles, se pueden utilizar los gráficos de crecimiento de la OMS desde el nacimiento hasta los 5 años de edad, después de los cuales se recomienda la Referencia de 2007 de la OMS.

- La mayoría de los lactantes nacidos PEG se recuperan de su déficit de talla y peso.

- El tratamiento con hormona de crecimiento beneficia el potencial de crecimiento en los niños nacidos PEG con talla baja.

- Se debería tomar en cuenta la función gonadal en ambos sexos, especialmente durante la pubertad.

- No se les debería permitir a los niños nacidos PEG ganar peso demasiado rápido o excesivamente en un esfuerzo para evitar el desarrollo de trastornos metabólicos.

- Un equipo multidisciplinario debería seguir de manera cuidadosa a los niños nacidos PEG.

- Se necesita un programa para la difusión médica y entrenamiento de los miembros del equipo multidisciplinario.

\section{Agradecimientos}

Agradecemos a Adriane de André CardosoDemartini por su apoyo en la preparación del manuscrito. Los servicios de redacción médica y la asistencia editorial fueron proporcionados por ACUMED ${ }^{\circledR}$ (Nueva York, N.Y.).

\section{Referencias}

1.- Ramakrishnan $U$ : Nutrition and low birth weight: from research to practice. Am J Clin Nutr 2004; 79: 17-21.

2.- United Nations Children's Fund 2003 State of the World's Children Report, 2003 [http://www.unicef.org/ sowc03/contents/pdf/SOWC03-eng.pdf] Accessed July $1,2010$.

3.- Clayton PE, Cianfarani S, Czernichow P, Johannsson $G$, Rapaport R, Rogol A: Management of the child born small for gestational age through to adulthood: a consensus statement of the International Societies of Pediatric Endocrinology and the Growth Hormone Research Society. J Clin Endocrinol Metab 2007; 92: 804-10.

4.- Barker DJ: Mothers, babies, and disease in later life. London: British Medical Journal Publishing Group; 1998.

5.- Hardin DS: Treatment of short stature and growth hormone deficiency in children with somatotropin (rDNA origin). Biologics 2008; 2: 655-61.

6.- Intrauterine growth retardation in newborn children [http://www.who.int/ceh/indicators/iugrnewborn.pdf] Accessed July 1, 2010. 
7.- Battaglia FC, Lubchenco LO: A practical classification of newborn infants by weight and gestational age. J Pediatr 1967; 71: 159-63.

8.- Williams RL, Creasy RK, Cunningham GC, Hawes WE, Norris FD, Tashiro M: Fetal growth and perinatal viability in California. Obstet Gynecol 1982; 59: 62432.

9.- Physical status: the use and interpretation of anthropometry. Report of a WHO Expert Committee. Technical Report Series No. 854 [http://www.who.int/ childgrowth/publications/physical_status/en/ Published 1995]. Accessed July 1, 2010.

10.- Lee PA, Chernausek SD, Hokken-Koelega AC, Czernichow $P$ : International Small for Gestational Age Advisory Board 2003 International Small for Gestational Age Advisory Board consensus development conference statement: management of short children born small for gestational age, April 24-October 1, 2001. Pediatrics 2003; 111: 1253-61.

11.- Saenger P, Czernichow P, Hughes I, Reiter EO: Small for gestational age: short stature and beyond. Endocr Rev 2007; 28: 219-51.

12.- Flores $H A$, Valverde $M G$, Islas GA: Peso al nacer de los niños y niñas. In: Flores HA, Martínez SH, eds. Prácticas de alimentación, estado de nutrición y cuidados a la salud en ninos menores de 2 años en México. México: Instituto Mexicano del Seguro Social; 2004.

13.- Centro Latinoamericano de Perinatología y Desarrollo Humano, OPS/OMS Boletín de Salud Perinatal 2000; 18: 7-9.

14.- The top 10 causes of death [http://www.who.int/mediacentre/factsheets/fs310/en/]. Updated October 2008. Accessed July 1, 2010.

15.- Mericq V, Ong KK, Bazaes $R$, et al: Longitudinal changes in insulin sensitivity and secretion from birth to age three years in small- and appropriate-for-gestational-age children. Diabetologia 2005; 48: 2609-14.

16.- Hokken-Koelega A, van Pareren Y, Arends N: Effects of growth hormone treatment on cognitive function and head circumference in children born small forgestational age. Horm Res 2005; 64 (Suppl 3): 95-9.

17.- Eriksson JG, Forsen T, Tuomilehto J, Jaddoe VW, Osmond C, Barker DJ: Effects of size at birth and childhood growth on the insulin resistance syndrome in elderly individuals. Diabetologia 2005; 45: 342-8.

18.- Eriksson JG, Forsen T, Tuomilehto J, Winter PD, Osmond C, Barker DJ: Catch-up growth in childhood and death from coronary heart disease: longitudinal study. BMJ 1999; 318: 427-31.

19.- Ballard JL, Novak KK, Driver M: A simplified score for assessment of fetal maturation of newly born infants. J Pediatr 1979; 95: 769-74.

20.- Fenton TR: A new growth chart for preterm babies: Babson and Benda's chart updated with recent data and a new format. BMC Pediatr 2003; 3: 13.

21.- Engstrom JL, Kavanaugh K, Meier PP, Boles E, Hernandez J, Wheeler D, Chuffo R: Reliability of in-bed weighing procedures for critically ill infants. Neonatal Netw 1995; 14: 27-33.

22.- Sutter K, Engstrom JL, Johnson TS, Kavanaugh K, Ifft $D L$ : Reliability of head circumference measurements in preterm infants. Pediatr Nurs 1997; 23: 485-90.

23.- Rosenberg SN, Verzo B, Engstrom JL, Kavanaugh $K$, Meier PP: Reliability of length measurements for preterm infants. Neonatal Netw 1993; 11: 23-7.

24.- Fomon SJ, Nelson SE: Size and growth. In: Fomon SJ. Nutrition of normal infants. St Louis, MO: Mosby; 1993; 49.

25.- Wollman H: Children born small for gestational age: definitions and etiology. In: Keiss W, Chernausek SD, Hokken-Koelega A, eds. 2009 Small for gestational age, causes and consequences. pediatric and adolescent medicine. Volume 13. Karger AG, Basel 2009; 1-10.

26.- González RP, Gómez RM, Castro RS, et al: A national birth weight distribution curve according to gestational age in Chile from 1993 to 2000. Rev Med Chile 2004; 132: 1155-65.

27.- Lejarraga H, Orfila G: Height and weight standards for Argentine children from birth to maturity. Arch Argent Pediatr 1987 (a); 85: 209-22.

28.- Lejarraga H, Morasso $M$ del C, Orfila G: Standards weight / age and weight / height for children under 6 years in primary care. Arch Argent Pediatr 1987 (b); 85: 69-76.

29.- Lejarraga H, del Pino M, Fano V, Caino S, Cole TJ: Growth references for weight and height for Argentinian girls and boys from birth to maturity: incorporation of data from the World Health Organisation from birth to 2 years and calculation of new percentiles and LMS values. Arch Argent Pediatr 2009; 107: 126-33.

30.- Rao SC, Tompkins J; World Health Organization: Growth curves for preterm infants. Early Hum Dev 2007; 83: 643-51.

31.- Karlberg J, Albertsson-Wikland K: Growth in full-term small-for-gestational-age infants: from birth to final height. Pediatr Res 1995; 38: 733-9.

32.- Albertsson-Wikland K, Boguszewski M, Karlberg $J$ : Children born small-forgestational age: postnatal growth and hormonal status. Horm Res 1995; 49: 7-13.

33.- Albertsson-Wikland K, Karlberg J: Postnatal growth of 
children born small for gestational age. Acta Paediatr Suppl 1997; 423: 193-5.

34.- Finken MJ, Dekker FW, de Zegher F, Wit JM: Dutch Project on Preterm and Small-for-Gestational-Age-19 Collaborative Study Group 2006. Long-term height gain of prematurely born children with neonatal growth restraint: parallellism with the growth pattern of short children born small for gestational age. Pediatrics 2006; 118: 640-3.

35.- Leger J, Garel C, Fjellestad-Paulsen A, Hassan M, Czernichow P: Human growth hormone treatment of short-stature children born small for gestational age: effecton muscle and adipose tissue mass during a 3 -year treatment period and after 1 year's withdrawal. J Clin Endocrinol Metab 1995; 83: 3512-6.

36.- Leger J, Levy-Marchal C, Bloch J, et al: Reduced final height and indications for early development of insulin resistance in a 20 year old population born with intra uterine growth retardation. BMJ 1997; 315: 341-7.

37.- Karlberg JP, Albertsson-Wikland K, Lam BC, Low $B C$ : The timing of early postnatal catch-up growth in normal, full-term infants born short for gestational age. Horm Res 1997; 48 (Suppl 1): 17-24.

38.- de Waal WJ, Hokken-Koelega AC, Stijnen T, de Muinck Keizer-Schrama SM, Drop SL: Endogenous and stimulated GH secretion, urinary GH excretion, and plasma IGF-I and IGF-II levels in prepubertal children with short stature after intrauterine growth retardation. The Dutch Working Group on Growth Hormone. Clin Endocrinol 1994; 41: 621-30.

39.- The WHO child growth standards [http://www.who.int/ childgrowth/standards/en/]. Accessed July 1, 2010.

40.- Growth reference data for 5-19 years [http://www.who. int/growthref/en/]. Accessed July 1, 2010.

41.- Gharib H, Cook DM, Saenger PH, et al; American Association of Clinical Endocrinologists Growth Hormone Task Force 2003: American Association of Clinical Endocrinologists medical guidelines for clinical practice for growth hormone use in adults and children-2003 update. Endocr Pract 2003, 9: 64-73.

42.- Genotropin (somatropin) injection CenterWatch Web site [http://www.centerwatch.com/drug-information/ fda-approvales/drugdetails.aspx?DrugID $=712]$. Accessed July 1, 2010.

43.- European Agency for the Evaluation of Medicinal Products: Committee for Proprietary Medicinal Products (CPMP) 2003 Norditropin. [http://www.ema.europa. eu/pdfs/human/referral/norditropin/347803en.pdf]. Published June 26, 2003. Accessed May 12, 2010.

44.- Argente J, Gracia R, Ibáñez L, et al; Spanish SGA
Working Group: Improvement in growth after two years of growth hormone therapy in very young children born small for gestational age and without spontaneous catch-up growth: results of a multicenter, controlled, randomized, open clinical trial. J Clin Endocrinol Metab 2007; 92: 3095-101.

45.- Lanes R, Plotnick LP, Lee PA: Sustained effect of human growth hormone therapy on children with intrauterine growth retardation. Pediatrics 1979; 63: 731-5.

46.- Dahlgren J, Wikland KA; Swedish Study Group for Growth Hormone Treatment: Final height in short children born small for gestational age treated with growth hormone. Pediatr Res 2005; 57: 216-22.

47.- Wilson TA, Rose SR, Cohen P, et al; Lawson Wilkins Pediatric Endocrinology Society Drug and Therapeutics Committee: Update of guidelines for the use of growth hormone in children: the Lawson Wilkins Pediatric Endocrinology Society Drug and Therapeutics Committee. J Pediatr 2003; 143: 415-21.

48.- Bergada I, Blanco M, Keselman A, Domene HM, Bergada $C$ : Growth hormone treatment in younger than six years of age short children born small for gestational age. Arch Argent Pediatr 2009; 107: 410-6.

49.- van Dijk M, Bannink EM, van Pareren $Y K$, Mulder $P G$, Hokken-Koelega AC: Risk factors for diabetes mellitus type 2 and metabolic syndrome are comparable for previously growth hormone-treated young adults born small for gestational age (SGA) and untreated short SGA controls. J Clin Endocrinol Metab 2007; 92: 1605 .

50.- Willemsen RH, van Dijk $M$, de Rijke YB, van Toorenenbergen AW, Mulder PG, Hokken-Koelega AC: Effect of growth hormone therapy on serum adiponectin and resistin levels in short, small-for-gestational-age children and associations with cardiovascular risk parameters. J Clin Endocrinol Metab 2007; 92: 117-23.

51.- Cutfield WS, Lindberg A, Rapaport R, Wajnrajch MP, Saenger P: Safety of growth hormone treatment in children born small for gestational age: the US trial and KIGS analysis. Horm Res 2006; 65 (Suppl 3): 153-9.

52.- Hales CN, Barker DJ, Clark PM, et al: Fetal and infant growth and impaired glucose tolerance at age 64. BMJ 1991; 303: 1019-22.

53.- Barker DJ, Osmond C, Golding J, Kuh D, Wadsworth $M E$ : Growth in utero, blood pressure in childhood and adult life, and mortality from cardiovascular disease. BMJ 1989; 298: 564-7.

54.- Barker DJ, Winter PD, Osmond C, Margetts B, Simmonds SJ: Weight in infancy and death from ischaemic heart disease. Lancet 1989; 2: 577-80. 
55.- Jaquet D, Gaboriau A, Czernichow P, Levy-Marchal $C$ : Insulin resistance early in adulthood in subjects born with intrauterine growth retardation. J Clin Endocrinol Metab 2000; 85: 1401-6.

56.- van Pareren $Y$, Mulder P, Houdijk M, Jansen M, Reeser M, Hokken-Koelega A: Effect of discontinuation of growth hormone treatment on risk factors for cardiovascular disease in adolescents born small for gestational age. J Clin Endocrinol Metab 2009; 88: 347-53.

57.- Chatelain P, Carrascosa A, Bona G, Ferrandez-Longas $A$, Sippell $W$ : Growth hormone therapy for short children born small for gestational age. Horm Res 2007; 68: 300-9.

58.- Czernichow P: Growth hormone treatment strategy for short children born small for gestational age. Horm Res 2004; 62 (Suppl 3): 137-40.

59.- Simon D, Leger J, Carel JC: Optimal use of growth hormone therapy for maximizing adult height in children born small for gestational age. Best Pract Res Clin Endocrinol Metab 2008; 22: 525-37.

60.- Bannink EM, van Doorn J, Mulder PG, HokkenKoelega $A C$ : Free/dissociable insulinlike growth factor (IGF)-I, not total IGF-I, correlates with growth response during growth hormone treatment in children born small for gestational age. J Clin Endocrinol Metab 2007; 92: 2992-3000.

61.- Ibáñez L, Ferrer A, Marcos MV, Hierro FR, de Zegher $F$ : Early puberty: rapid progression and reduced final height in girls with low birth weight. Pediatrics 2000; 106: E72.

62.- Lazar L, Pollak U, Kalter-Leibovici O, Pertzelan A, Phillip M: Pubertal course ofpersistently short children born small for gestational age (SGA) compared with idiopathic short children born appropriate for gestational age (AGA). Eur J Endocrinol 2003; 149: 425-32.

63.- Lienhardt A, Carel JC, Preux PM, Coutant R, Chaussain JL: Amplitude of pubertal growth in short stature children with intrauterine growth retardation. Horm Res 2002; 57 (Suppl 2): 88-94.

64.- Veening MA, van Weissenbruch MM, Roord JJ, de Delemarre-van Waal HA: Pubertal development in children born small for gestational age. J Pediatr Endocrinol Metab 2004; 17: 1497-505.

65.- Persson I, Ahlsson F, Ewald U, et al: Influence of perinatal factors on the onset of puberty in boys and girls: implications for interpretation of link with risk of long term diseases. Am J Epidemiol 1999; 150: 747-55.

66.- Albertsson-Wikland K, Karlberg J: Natural growth in children born small for gestational age with and wi- thout catch-up growth. Acta Paediatr Suppl 1994; 399 : 64-70.

67.- Ghirri P, Bernardini M, Vuerich M, et al: Adrenarche, pubertal development, age at menarche and final height of full-term, born small for gestational age (SGA) girls. Gynecol Endocrinol 2001; 15: 91-7.

68.- Boonstra V, van Pareren Y, Mulder P, Hokken-Koelega $A$ : Puberty in growth hormone-treated children born small for gestational age (SGA). J Clin Endocrinol Metab 2003; 88: 5753-8.

69.- Vicens-Calvet E, Espadero RM, Carrascosa A: Longitudinal study of the pubertal growth spurt in children born small for gestational age without postnatal catchup growth. J Pediatr Endocrinol Metab 2002; 15: 3818.

70.- Cara JF, Rosenfield RL: Insulin-like growth factor I and insulin potentiate luteinizing hormone-induced androgen synthesis by rat ovarian thecal-interstitial cells. Endocrinology 1998; 123: 733-9.

71.- Bhargava SK, Ramji S, Srivastava U, et al: Growth and sexual maturation of low birth weight children: a 14 year follow up. Indian Pediatr 1995; 32: 963-70.

72.- Hernández MI, Mericq V: Impact of being born small for gestational age on onset and progression of puberty. Best Pract Res Clin Endocrinol Metab 2008; 22: 46376.

73.- Ibáñez L, Jiménez $R$, de Zegher F: Early puberty-menarche after precocious pubarche: relation to prenatal growth. Pediatrics 2006; 117: 117-21.

74.- Koziel S, Jankowska EA: Effect of low versus normal birthweight on menarche in 14-year-old Polish girls. J Paediatr Child Health 2002; 38: 268-71.

75.- Sloboda DM, Hart R, Doherty DA, Pennell CE, Hickey $M$ : Age at menarche: influences of prenatal and postnatal growth. J Clin Endocrinol Metab 2007; 92: 46-50.

76.- Ibáñez L, Potau N, Francois I, de Zegher F: Precocious pubarche, hyperinsulinism, and ovarian hyperandrogenism in girls: relation to reduced fetal growth. J Clin Endocrinol Metab 1998; 83: 3558-62.

77.- Job JC, Rolland A: Natural history of intrauterine growth retardation: pubertal growth and adult height. Arch Fr Pediatr 1986; 43: 301-6.

78.- Ibáñez L, de Zegher F, Potau N: Anovulation after precocious pubarche: early markers and time course in adolescence. J Clin Endocrinol Metab 1999; 84: 26915.

79.- Ibáñez L, de Zegher F: Puberty after prenatal growth restraint. Horm Res 2006; 65 (Suppl 3): 112-5.

80.- Jaquet D, Leger J, Chevenne D, Czernichow P, LevyMarchal C: Intrauterine growth retardation predisposes 
to insulin resistance but not to hyperandrogenism in young women. J Clin Endocrinol Metab 1999; 84: 3945-9.

81.- Bazaes RA, Alegría A, Pittaluga E, Avila A, Iñiguez $G$, Mericq $V$ : Determinants of insulin sensitivity and secretion in very-low-birth-weight children. J Clin Endocrinol Metab 2004; 89: 1267-72.

82.- Boonstra VH, Mulder PG, de Jong FH, HokkenKoelega $A C$ : Serum dehydroepiandrosterone sulfate levels and pubarche in short children born small for gestational age before and during growth hormone treatment. J Clin Endocrinol Metab 2004; 89: 712-7.

83.- Eyzaguirre FC, Bancalari R, Youlton R, et al: Precocious pubarche: experience in 173 cases. Rev Med Chile 2009; 137: 31-8.

84.- Hernández MI, Martínez A, Capurro T, et al: Comparison of clinical, ultrasonographic, and biochemical differences at the beginning of puberty in healthy girls born either small for gestational age or appropriate for gestational age: preliminary results. J Clin Endocrinol Metab 2006; 91: 3377-81.

85.- Ibáñez L, Ferrer A, Ong K, Amin R, Dunger D, de Zegher $F$ : Insulin sensitization early after menarche prevents progression from precocious pubarche to polycystic ovary syndrome. J Pediatr 2004; 144: 23-9.

86.- Ibáñez L, Ong K, Valls C, Marcos MV, Dunger DB, de Zegher $F$ : Metformin treatment to prevent early puberty in girls with precocious pubarche. J Clin Endocrinol Metab 2006; 91: 2888-91.

87.- Main KM, Jensen RB, Asklund C, Høi-Hansen CE, Skakkebaek NE: Low birth weight and male reproductive function. Horm Res 2006, 65 (Suppl 3): 116-22.

88.- Boisen KA, Kaleva M, Main KM, et al: Difference in prevalence of congenital cryptorchidism in infants between two Nordic countries. Lancet 2004; 363: 1264-9.

89.- Boisen KA, Chellakooty M, Schmidt IM, et al: Hypospadias in a cohort of 1072 Danish newborn boys: prevalence and relationship to placental weight, anthropometrical measurements at birth, and reproductive hormone levels at three months of age. J Clin Endocrinol Metab 2005; 90: 4041-6.

90.- Hughes IA, Northstone K, Golding J: Reduced birth weight in boys with hypospadias: an index of androgen dysfunction? Arch Dis Child Fetal Neonatal Ed 2002; 87: F150-1.

91.- Pierik FH, Burdorf A, Deddens JA, Juttmann RE, Weber RF: Maternal and paternal risk factors for cryptorchidism and hypospadias: a case-control study in newborn boys. Environ Health Perspect 2004; 112: 1570-6.
92.- Cicognani A, Alessandroni R, Pasini A, et al: Low birth weight for gestational age and subsequent male gonadal function. J Pediatr 2002; 141: 376-9.

93.- Jensen RB, Vielwerth $S$, Larsen T, Greisen $G$, Veldhuis $J$, Juul A: Pituitary-gonadal function in adolescent males born appropriate or small for gestational age with or without intrauterine growth restriction. J Clin Endocrinol Metab 2007; 92: 1353-7.

94.- Dunger DB, Ong KK: Babies born small for gestational age: insulin sensitivity and growth hormone treatment. Horm Res 2005; 64 (Suppl 3): 58-65.

95.- Mericq V, Medina P, Kakarieka E, Márquez L, Johnson $M C$, Iñiguez $G$ : Differences in expression and activity of 11 beta-hydroxysteroid dehydrogenase type 1 and 2 in human placentas of term pregnancies according to birth weight and gender. Eur J Endocrinol 2009; 161: 419-25.

96.- Seckl JR, Holmes MC: Mechanisms of disease: glucocorticoids, their placental metabolism and fetal 'programming' of adult pathophysiology. Nat Clin Pract Endocrinol Metab 2007; 3: 479-88.

97.- Phillips DI, Barker DJ, Fall CH, et al: Elevated plasma cortisol concentrations: a link between low birth weight and the insulin resistance syndrome? J Clin Endocrinol Metab 1998; 83: 757-60.

98.- Hattersley AT, Tooke JE: The fetal insulin hypothesis: an alternative explanation of the association of low birthweight with diabetes and vascular disease. Lancet 1999; 353: 1789-92.

99.- Soto N, Bazaes RA, Peña V, et al: Insulin sensitivity and secretion are related to catch-up growth in smallfor-gestational-age infants at age 1 year: results from a prospective cohort. J Clin Endocrinol Metab 2003; 88 : 3645-50.

100.- Veening MA, Van Weissenbruch MM, Delemarre-Van De Waal HA: Glucose tolerance, insulin sensitivity, and insulin secretion in children born small for gestational age. J Clin Endocrinol Metab 2002; 87: 4657-61.

101.- Veening MA, van Weissenbruch MM, Heine RJ, Delemarre-van de Waal HA: Betacell capacity and insulin sensitivity in prepubertal children born small for gestational age: influence of body size during childhood. Diabetes 2003; 52: 1756-60.

102.- Jaquet D, Deghmoun S, Chevenne D, Collin D, Czernichow $P$, Lévy-Marchal C: Dynamic change in adiposity from fetal to postnatal life is involved in the metabolic syndrome associated with reduced fetal growth. Diabetologia 2005; 48: 849-55.

103.- Chatelain P: Children born with intrauterine growth retardation (IUGR) or small for gestational age: long- 
term growth and metabolic consequences. Endocr Rev 2000; 33: 33-6.

104.- Murtaugh MA, Jacobs DR Jr, Morán A, Steinberger J, Sinaiko AR: Relation of birth weight to fasting insulin, insulin resistance, and body size in adolescence. Diabetes Care 2003; 26: 187-92.

105.- Carel JC, Chatelain P, Rochiccioli P, Chaussain JL: Improvement in adult height after growth hormone treatment in adolescents with short stature born small for gestational age: results of a randomized controlled study. J Clin Endocrinol Metab 2003; 88: 1587-93.

106.- Cutfield WS, Jackson WE, Jefferies C, et al: Reduced insulin sensitivity during growth hormone therapy for short children born small for gestational age. J Pediatr 2003; 142: 113-6.

107.- Cutfield WS, Wilton P, Bennmarker H, et al: Incidence of diabetes mellitus and impaired glucose tolerance in children and adolescents receiving growth-hormone treatment. Lancet 2000; 355: 610-3.

108.- de Zegher F, Ong K, van Helvoirt M, Mohn A, Woods K, Dunger D: High-dose growth hormone $(\mathrm{GH})$ treatment in non-GH-deficient children born small for gestational age induces growth responses related to pretreatment $\mathrm{GH}$ secretion and associated with a reversible decrease in insulin sensitivity. J Clin Endocrinol Metab 2002; 87: 148-51.

109.- Hofman PL, Cutfield WS, Robinson EM, et al: Insulin resistance in short children with intrauterine growth retardation. J Clin Endocrinol Metab 1997; 82: 402-6.

110.- Hokken-Koelega A, van Pareren Y, Arends N, Boonstra $V$ : Efficacy and safety of long-term continuous growth hormone treatment of children born small for gestational age. Horm Res 2004; 62 (Suppl 3): 149-54.

111.- Willemsen RH, Willemsen SP, Hokken-Koelega AC: Longitudinal changes in insulin sensitivity and body composition of small-for-gestational-age adolescents after cessation of growth hormone treatment. J Clin Endocrinol Metab 2008; 93: 3449-54.

112.- Willemsen RH, de Kort $S W$, van der Kaay DC: Independent effects of prematurity on metabolic and cardiovascular risk factors in short small-for-gestational-age children. J Clin Endocrinol Metab 2008; 93: 452-8. 